\title{
Spectral Properties of Random and Deterministic CMV Matrices
}

\author{
M. Stoiciu * \\ Department of Mathematics and Statistics, Williams College, Williamstown, MA 01267, USA
}

\begin{abstract}
The CMV matrices are unitary analogues of the discrete one-dimensional Schrödinger operators. We review spectral properties of a few classes of CMV matrices and describe families of random and deterministic CMV matrices which exhibit a transition in the distribution of their eigenvalues.
\end{abstract}

Keywords and phrases: spectral theory, distribution of eigenvalues, CMV matrices

Mathematics Subject Classification: 81Q10, 47B06, 42C05

\section{Introduction}

Recent developments in the theory of orthogonal polynomials on the unit circle have emphasized the importance of a class of unitary matrices called CMV matrices. These matrices can be regarded as the unitary analogues of Jacobi matrices, hence one expects many parallels between the spectral theory of CMV matrices and of Jacobi matrices/discrete Schrödinger operators. In this paper we review certain spectral properties (more precisely, the distribution of the eigenvalues) of some classes of CMV matrices. We describe families random and deterministic CMV matrices which exhibit a transition in the distribution of their eigenvalues.

The study of random matrices provides answers to questions regarding localization and transport, which are central in condensed matter physics and especially in the field of quantum mechanics of disordered media. A very suitable model for the study of these phenomena is a random Schrödinger operator introduced and analyzed in [3]; this mathematical object is now called the Anderson model. For this work, Philip W. Anderson was awarded the Nobel Prize in Physics in 1977. The first rigorous mathematical results on this topic appeared in the second half of the 1970s and many important discoveries followed. However, several important questions regarding the transition between the extended regime (which exhibits transport) and the localized regime (where there is no transport) are still open. A relevant method to characterize the extended states regime is the analysis of the eigenvalue statistics. The transition from the extended to the localized states is reflected in different level statistics: we observe repulsion between the eigenvalues in the extended states regime and non-correlation (Poisson statistics) of the eigenvalues in the localized regime.

\footnotetext{
*Corresponding author. E-mail: mstoiciu@williams.edu
} 
The one-dimensional discrete Schrödinger operator (from the Anderson model) is a special type of Jacobi matrix; thus, the spectral theory for discrete one-dimensional Schrödinger operators is intimately connected to the theory of orthogonal polynomials on the real line. Following this analogy, we can consider probability measures on the unit circle, the associated orthogonal polynomials on the unit circle and the corresponding unitary operators. These operators, called CMV, (see the monographs [25] and $[26])$, are the unitary analogues of the Jacobi operators.

In Section 2 we will define random CMV matrices that are the unitary analogues of the random onedimensional discrete Schrödinger operator from the Anderson model and we will describe their eigenvalue statistics. In particular, we will show that the random CMV matrices with random decaying coefficients exhibit a transition in the distribution of their eigenvalues, as described in [17]. As conjectured for random Schrödinger operators, the random CMV matrices exhibit a transition in the eigenvalue statistics from no correlation (Poisson) to strong repulsion ("clock"), while at the transitional point the eigenvalues are distributed according to the circular beta ensemble of the random matrix theory [19].

We continue in Section 3 with a review of some of the spectral properties of the CMV matrices associated to the a family of singular continuous measures on the unit circle generated by McMullen's three circle family. These deterministic matrices also exhibit a transition in their eigenvalue distribution towards the "clock" distribution, as their spectral measure approaches an absolutely continuous measure.

\section{Spectral Properties of Random CMV Matrices}

Many questions that arise in the spectral theory of the random CMV matrices are motivated by similar questions for random Schrödinger operators. Furthermore, a good number of techniques used in the analysis of random self-adjoint operators (random Jacobi and Schrödinger operators) are easily applied to the unitary case (to random CMV matrices). Hence we will start this section with an overview of the known results on the eigenvalue distribution of random Schrödinger operators.

Philip W. Anderson's ground-breaking work [3], on the transport properties of disordered systems, was a major breakthrough in the quantum mechanics of random media. Anderson received the Nobel Prize in Physics in 1977 "for fundamental theoretical investigations of the electronic structure of magnetic and disordered systems". Referring to the phenomenon now called "Anderson localization", he said in his Nobel Lecture that: "It has yet to receive adequate mathematical treatment, and one has to resort to the indignity of numerical simulations to settle even the simplest questions about it".

The first rigorous results of the Anderson localization were obtained in mid-seventies by Russian mathematicians $([13,21])$. Other proofs were obtained later $([1,2,5,11])$, showing that the mathematics behind the Anderson localization is extremely rich and complex. Most of the current mathematical proofs of the Anderson Localization show that a stochastic Hamiltonian with independent identically distributed random potential has, with probability 1, pure point spectrum and exponentially decaying eigenfunctions. However, there is a debate on what localization actually means (see, for example, [8]), and one alternative is to analyze the level statistics of the eigenvalues of the random Hamiltonian.

The first result on the eigenvalue statistics of the spectrum of the one-dimensional Schrödinger operators was obtained by Molchanov in [22]. Molchanov considered a one-dimensional continuous Schrödinger operator

$$
H(\omega)=-\frac{d^{2}}{d t^{2}}+q(t, \omega)
$$

where the random potential $q(t, \omega)=F\left(x_{t}(\omega)\right)$, is obtained by applying a smooth nonflat Morse function $F: K \rightarrow \mathbb{R}$ to the Brownian motion $x_{t}$ on a compact manifold $K$.

Settling a controversy in the mathematical physics literature, Molchanov showed that there is no repulsion between the energy levels of the finite-volume random Schrödinger operator, as the volume approaches infinity. More precisely, if $H_{V}=H_{V}(\omega)$ is the random one-dimensional Schrödinger operator on $L^{2}(-V, V)$ with Dirichlet boundary conditions and $N_{V}(I)$ is the number of eigenvalues of the operator $H_{V}$ situated in the interval $I$, then, for any fixed $a_{1}<b_{1} \leq a_{2}<b_{2} \leq \cdots \leq a_{n}<b_{n}$ and any nonnegative 
integers $k_{1}, k_{2}, \ldots, k_{n}$,

$$
\begin{aligned}
\lim _{V \rightarrow \infty} & \mathbb{P}\left(N_{V}\left(E_{0}+\frac{a_{1}}{2 V}, E_{0}+\frac{b_{1}}{2 V}\right)=k_{1}, \ldots, N_{V}\left(E_{0}+\frac{a_{n}}{2 V}, E_{0}+\frac{b_{n}}{2 V}\right)=k_{n}\right) \\
& =e^{-\left(b_{1}-a_{1}\right) n\left(E_{0}\right)} \frac{\left(\left(b_{1}-a_{1}\right) n\left(E_{0}\right)\right)^{k_{1}}}{k_{1} !} \cdots e^{-\left(b_{n}-a_{n}\right) n\left(E_{0}\right)} \frac{\left(\left(b_{n}-a_{n}\right) n\left(E_{0}\right)\right)^{k_{n}}}{k_{n} !}
\end{aligned}
$$

This means that the local statistical distribution of the eigenvalues of the operator $H_{V}$ (rescaled near the point $\left.E_{0}\right)$ converges, as $V \rightarrow \infty$, to the Poisson point process with intensity measure $n\left(E_{0}\right) d x$, where $d x$ denotes the Lebesgue measure and $n\left(E_{0}\right)$ is the density of states at $E_{0}$. Hence, Molchanov's result shows that there is no local correlation between the energy levels of the random one-dimensional Schrödinger operator.

The next challenge for mathematical physicists was to see whether a similar result holds for multidimensional Schrödinger operators. A positive answer to this question was given by Minami in [20], who showed that near energies where Anderson localization is expected (more precisely, in regimes where the Anderson localization holds via [1]), there is no correlation between the eigenvalues of the Anderson tight-binding model.

In order to describe Minami's result, consider $H=-\Delta+V_{\omega}$, the standard Anderson tight-binding model acting in $l^{2}\left(\mathbb{Z}^{d}\right)$, and let $H^{\Lambda}=\chi_{\Lambda} H \chi_{\Lambda}$ be the truncated Schrödinger operator corresponding to the hypercube $\Lambda \subset \mathbb{Z}^{d}$. Also, let $G^{\Lambda}(z)=\left(H^{\Lambda}-z\right)^{-1}$ be the resolvent associated to $H^{\Lambda}$. If $E_{1}(\Lambda) \leq$ $E_{2}(\Lambda) \leq \cdots \leq E_{n}(\Lambda)$ are the eigenvalues of $H^{\Lambda}$, then the integrated density of states at $E$ is given by

$$
N(E)=\lim _{\Lambda \uparrow \mathbb{Z}^{d}} \frac{1}{|\Lambda|} \#\left\{j, E_{j}(\Lambda) \leq E\right\}
$$

and the density of states at $E$ by

$$
n(E)=d N(E) / d E
$$

Note that if $n(E)>0$, then the average spacing of the eigenvalues near the energy $E$ is of order $|\Lambda|^{-1}$. Consider the rescaled spectrum (near $E$ ):

$$
\xi_{j}(\Lambda, E)=|\Lambda|\left(E_{j}(\Lambda)-E\right)
$$

and the corresponding point process

$$
\xi(\Lambda, E)=\sum_{j} \delta_{\xi_{j}(\Lambda, E)}
$$

Minami proved in [20] that, under suitable conditions:

$$
\left.\xi(\Lambda, E) \stackrel{\Lambda \uparrow \mathbb{Z}^{d}}{\longrightarrow} \text { (Poisson point process of intensity } n(E)\right)
$$

This means exactly that there is no correlation between the eigenvalues of $H^{\Lambda}$ if $\Lambda$ is large.

We should mention here that one of the main ingredients of the proof is the following technical lemma:

$$
\mathbb{E}\left[\operatorname{det}\left(\begin{array}{l}
\operatorname{Im} G^{\Lambda}(z ; x, x) \operatorname{Im} G^{\Lambda}(z ; x, y) \\
\operatorname{Im} G^{\Lambda}(z ; y, x) \operatorname{Im} G^{\Lambda}(z ; y, y)
\end{array}\right)\right] \leq C
$$

where the constant $C$ depends only on the distribution of the potential $V=V_{\omega}$. The proof of the result relies on a technique called the "Minami trick". It is worth mentioning that this technique encodes a spectacular and somewhat mysterious cancelation that has found applications to other questions in mathematical physics; see for example $[4,6,14,15]$.

A 2003 paper of Cantero, Moral, and Velázquez [7] emphasized the importance of a new class of unitary random matrices called now CMV matrices. These matrices are intimately connected with the orthogonal 
polynomials on the unit circle (see the monographs [25] and [26] on the theory of orthogonal polynomials on the unit circle and the review article [28]).

The CMV matrix is a five-diagonal matrix realization for the unitary operator $f(z) \rightarrow z f(z)$ on $L^{2}(\mathbb{T} ; \mu)$, where $\mu$ is a non-trivial probability measure on the unit circle $\mathbb{T}$ (we call a measure nontrivial if it is not supported on finitely many points). For any such measure $\mu$ we can apply the GramSchmidt procedure to the set of polynomials $\left\{1, z, z^{2}, \ldots\right\} \in L^{2}(\mathbb{T}, \mu)$ and get the set of monic orthogonal polynomials $\left\{\Phi_{0}(z, d \mu), \Phi_{1}(z, d \mu), \Phi_{2}(z, d \mu), \ldots\right\} \in L^{2}(\mathbb{T} ; \mu)$.

These polynomials obey the recurrence relation

$$
\Phi_{k+1}(z, d \mu)=z \Phi_{k}(z, d \mu)-\bar{\alpha}_{k} \Phi_{k}^{*}(z, d \mu) \quad k \geq 0
$$

where, for $\Phi_{k}(z)=\sum_{j=0}^{k} b_{j} z^{j}$, the reversed polynomial $\Phi_{k}^{*}(z)$ is defined by $\Phi_{k}^{*}(z)=\sum_{j=0}^{k} \bar{b}_{k-j} z^{j}$. The recurrence coefficients $\left\{\alpha_{n}\right\}_{n \geq 0}$ are called Verblunsky coefficents; they are complex numbers of absolute value $<1$.

If we apply the Gram-Schmidt algorithm to the sequence $\left\{1, z, z^{-1}, z^{2}, z^{-2}, \ldots\right\}$ we get the set of functions $\left\{\chi_{0}(z), \chi_{1}(z), \chi_{2}(z), \ldots\right\}$, which is an orthonormal basis of $L^{2}(\mathbb{T} ; \mu)$. The CMV matrix associated to the measure $\mu$ is the matrix representation of the operator $f(z) \rightarrow z f(z)$ on $L^{2}(\mathbb{T} ; \mu)$. It has the form:

$$
\mathcal{C}=\left(\begin{array}{cccccc}
\bar{\alpha}_{0} & \bar{\alpha}_{1} \rho_{0} & \rho_{1} \rho_{0} & 0 & 0 & \ldots \\
\rho_{0} & -\bar{\alpha}_{1} \alpha_{0} & -\rho_{1} \alpha_{0} & 0 & 0 & \ldots \\
0 & \bar{\alpha}_{2} \rho_{1} & -\bar{\alpha}_{2} \alpha_{1} & \bar{\alpha}_{3} \rho_{2} & \rho_{3} \rho_{2} & \ldots \\
0 & \rho_{2} \rho_{1} & -\rho_{2} \alpha_{1} & -\bar{\alpha}_{3} \alpha_{2} & -\rho_{3} \alpha_{2} & \ldots \\
0 & 0 & 0 & \bar{\alpha}_{4} \rho_{3} & -\bar{\alpha}_{4} \alpha_{3} & \ldots \\
\ldots & \ldots & \ldots & \ldots & \ldots & \ldots
\end{array}\right)
$$

where $\rho_{k}=\sqrt{1-\left|\alpha_{k}\right|^{2}}$.

Any CMV matrix $\mathcal{C}=\mathcal{C}\left(\alpha_{0}, \alpha_{1}, \alpha_{2}, \cdots\right)$ can be written as

$$
\mathcal{C}=\mathcal{L} \mathcal{M}
$$

where $\mathcal{L}$ and $\mathcal{M}$ are the tri-diagonal matrices

$$
\mathcal{L}=\left(\begin{array}{cccc}
\Theta_{0} & & & \\
& \Theta_{2} & & \\
& & \Theta_{4} & \\
& & & \ddots
\end{array}\right) \quad \mathcal{M}=\left(\begin{array}{cccc}
1 & & & \\
& \Theta_{1} & & \\
& & \Theta_{3} & \\
& & & \ddots
\end{array}\right)
$$

where, for any $k, \Theta_{k}=\Theta_{k}\left(\alpha_{k}\right)$ is the $2 \times 2$ matrix defined by

$$
\Theta_{k}=\left(\begin{array}{cc}
\bar{\alpha}_{k} & \rho_{k} \\
\rho_{k} & -\alpha_{k}
\end{array}\right), \quad \rho_{k}=\sqrt{1-\left|\alpha_{k}\right|^{2}}
$$

Note that the Jacobi matrices obtained in a similar way for orthogonal polynomials on the real line are tri-diagonal matrices. As in the case of orthogonal polynomials on the real line, an important connection between CMV matrices and monic orthogonal polynomials is

$$
\Phi_{n}(z)=\operatorname{det}\left(z I-\mathcal{C}^{(n)}\right)
$$

where $\mathcal{C}^{(n)}$ is the upper left $n \times n$ corner of $\mathcal{C}$. This immediately implies that the zeros of the polynomial $\Phi_{n}(z)$ are the eigenvalues of the matrix $\mathcal{C}^{(n)}$, a result which shows one of the many connections between approximation theory and spectral theory.

If $\left|\alpha_{n-1}\right|=1$, then the CMV matrix decouples between $(n-1)$ and $n$. The upper left corner is an $(n \times n)$ unitary matrix

$$
\mathcal{C}^{(n)}=\mathcal{C}_{\left\{\alpha_{0}, \alpha_{1}, \ldots, \alpha_{n-1}\right\}}^{(n)}
$$


Random CMV matrices and the statistical distribution of their eigenvalues is investigated in [29]. The CMV matrix $\mathcal{C}_{\left\{\alpha_{0}, \alpha_{1}, \ldots, \alpha_{n-1}\right\}}^{(n)}$ is randomized by taking independent identically distributed random variables $\alpha_{0}, \alpha_{1}, \ldots, \alpha_{n-2}$. The last variable, $\alpha_{n-1}$ is chosen to be uniformly distributed on the unit circle. As in the case of random Schrödinger operators, for various classes of $n \times n$ random CMV matrices, the local statistical distribution of the eigenvalues of these matrices converges (as $n \rightarrow \infty)$ to the Poisson distribution. This fact indicates that, as $n$ gets large, there is no local correlation between these eigenvalues, as the following theorem shows:

Theorem 2.1 (1.1. in [29]). Consider the random $C M V$ matrices $\mathcal{C}^{(n)}=\mathcal{C}_{\left\{\alpha_{0}, \alpha_{1}, \ldots, \alpha_{n-1}\right\}}^{(n)}$, where $\alpha_{0}, \alpha_{1}$, $\ldots, \alpha_{n-2}$ are i.i.d. random variables distributed uniformly in a disk of radius $r<1$, and $\alpha_{n-1}$ is another random variable independent of the previous ones and uniformly distributed on the unit circle.

Consider the space $\Omega_{n}=\left\{\alpha=\left(\alpha_{0}, \alpha_{1}, \ldots, \alpha_{n-2}, \alpha_{n-1}\right) \in D(0, r) \times D(0, r) \times \cdots \times D(0, r) \times \mathbb{T}\right\}$ with the probability measure $\mathbb{P}_{n}$ obtained by taking the product of the uniform Lebesgue measures on each $D(0, r)$ and on $\mathbb{T}$. Fix a point $e^{i \theta_{0}} \in \mathbb{T}$ and let $\zeta^{(n)}$ be the point process defined by $\zeta^{(n)}=\sum_{k=1}^{n} \delta_{z_{k}}$, where $\left\{z_{1}, z_{2}, \ldots, z_{n}\right\}$ are the eigenvalues of the matrix $\mathcal{C}^{(n)}$ (each eigenvalue $z_{i}$ depends on $\alpha_{0}, \alpha_{1}, \ldots, \alpha_{n-1}$ ).

Then, on a fine scale (of order $\frac{1}{n}$ ) near $e^{i \theta_{0}}$, the point process $\zeta^{(n)}$ converges to the Poisson point process with intensity measure $\frac{d \theta}{2 \pi}$ (the normalized Lebesgue measure on the unit circle). This means that for any fixed $a_{1}<b_{1} \leq a_{2}<b_{2} \leq \cdots \leq a_{m}<b_{m}$ and any nonnegative integers $k_{1}, k_{2}, \ldots, k_{m}$, we have

$$
\begin{aligned}
\mathbb{P}_{n}\left(\zeta^{(n)}\left(e^{i\left(\theta_{0}+\frac{2 \pi a_{1}}{n}\right)}, e^{i\left(\theta_{0}+\frac{2 \pi b_{1}}{n}\right)}\right)=k_{1}, \ldots, \zeta^{(n)}\left(e^{i\left(\theta_{0}+\frac{2 \pi a_{m}}{n}\right)}, e^{i\left(\theta_{0}+\frac{2 \pi b_{m}}{n}\right)}\right)=k_{m}\right) \\
\longrightarrow e^{-\left(b_{1}-a_{1}\right)} \frac{\left(b_{1}-a_{1}\right)^{k_{1}}}{k_{1} !} \cdots e^{-\left(b_{m}-a_{m}\right)} \frac{\left(b_{m}-a_{m}\right)^{k_{m}}}{k_{m} !}
\end{aligned}
$$

as $n \rightarrow \infty$.

The result is similar to the ones obtained for random Schrödinger operators by Molchanov and Minami $($ see $[20,22])$. The proofs use the same road map towards obtaining the Poisson distribution in the limit:

1. uniform estimates on the resolvent

2. control of the eigenfunctions

3. decoupling of the random matrix into smaller identical blocks

4. proving that the smaller blocks cannot contribute two or more eigenvalues in given intervals

5. obtaining the Poisson distribution as a limit of Bernoulli distributions

The uniform estimates on the resolvent are obtained using the fractional moments of the coefficients of the resolvent and originates from [1] and its successor [2]. The bounds on the resolvent are used to prove that the eigenfunctions of the random matrix $\mathcal{C}_{\alpha}^{(n)}$ are exponentially localized with probability 1.

The exponential localization allows the decoupling of the CMV matrix $\mathcal{C}^{(n)}=\mathcal{C}_{\alpha}^{(n)}$ into the direct sum $\sum_{p} \tilde{\mathcal{C}}_{p}^{(n)}$ of CMV matrices of smaller size, without changing the overall eigenvalue distribution. This shows that the limiting eigenvalue point process is infinitely divisible.

The next step is to prove that each small matrix $\tilde{\mathcal{C}}_{p}^{(n)}$ contributes at most one eigenvalue in the interval of size $\frac{1}{n}$. This follows from the fact that for any $e^{i \theta} \in \mathbb{T}$ and any interval $I_{n}$ of size $\frac{1}{n}$ near $e^{i \theta}$ the following "Minami-type" estimate holds:

$$
\mathbb{P}_{n}\left(\zeta^{(n, p)}\left(I_{n}\right) \geq 2\right)=O\left([\ln n]^{-2}\right) \quad \text { as } \quad n \rightarrow \infty
$$

which implies

$$
\sum_{p=1}^{[\ln n]} \mathbb{P}_{n}\left(\zeta^{(n, p)}\left(I_{n}\right) \geq 2\right) \longrightarrow 0 \quad \text { as } \quad n \rightarrow \infty
$$


Hence an interval of size $\frac{1}{n}$ contains $k$ eigenvalues if and only if exactly $k$ of the matrices $\tilde{\mathcal{C}}_{1}^{(n)}, \tilde{\mathcal{C}}_{2}^{(n)}, \ldots$ contribute one eigenvalue in the interval $I_{n}$. This is precisely a Bernoulli distribution and the limit as $n \rightarrow \infty$ is a Poisson distribution, which allows us to conclude that the limit $\zeta^{(n)}$ is a Poisson point process.

The previous theorem shows that if the coefficients $\alpha_{k}$ are uniformly distributed on a disk of radius $r<1$, then the eigenvalues of the corresponding CMV matrix $\mathcal{C}^{(n)}$ are not locally correlated for large $n$. A natural question is whether this result can be extended to different types of distributions for the coefficients $\alpha_{k}$. It turns out that the rotation invariance was critical in several places, but one can allow distributions that are singular with respect to the Lebesgue measure. Thus, if for $r \in(0,1)$, one takes $C(0, r)$ to be the circle of radius $r$ centered at the origin, one gets:

Theorem 2.2 (3.2 in [30]). Consider the random $C M V$ matrices $\mathcal{C}^{(n)}=\mathcal{C}_{\left\{\alpha_{0}, \alpha_{1}, \ldots, \alpha_{n-1}\right\}}^{(n)}$ where $\alpha_{0}, \alpha_{1}$, $\ldots, \alpha_{n-2}$ are i.i.d. random variables distributed uniformly in a circle of radius $r<1$, and $\alpha_{n-1}$ is another random variable independent of the previous ones and uniformly distributed on the unit circle.

Consider the space $\Omega_{n}=\left\{\alpha=\left(\alpha_{0}, \alpha_{1}, \ldots, \alpha_{n-2}, \alpha_{n-1}\right) \in C(0, r) \times C(0, r) \times \cdots \times C(0, r) \times \mathbb{T}\right\}$ with the probability measure $\mathbb{P}_{n}$ obtained by taking the product of the uniform (one-dimensional Lebesgue) measures on each $C(0, r)$ and on the unit circle $\mathbb{T}$. Fix a point $e^{i \theta_{0}} \in \mathbb{T}$ and let $\zeta^{(n)}$ be the point process obtained from the eigenvalues of the truncated $C M V$ matrix $\mathcal{C}^{(n)}$.

Then, on a fine scale, (of order $\frac{1}{n}$ ) near $e^{i \theta_{0}}$, the point process $\zeta^{(n)}$ converges to the Poisson point process with intensity measure $\frac{d \theta}{2 \pi}$ (the normalized Lebesgue measure on the unit circle).

The transition Poisson-Clock for the eigenvalue statistics of random CMV matrices with decaying coefficients is investigated in [17]. This transition is obtained as we change the rate of decay of the random coefficients. As the eigenvalue statistics changes from Poisson to Clock, it passes through a continuum of critical distributions given by the limiting circular $\beta$ ensemble from random matrix theory $(0<\beta<\infty)$. The constant $\beta$ is the inverse temperature corresponding to the log-gas from statistical mechanics $[10,19]$. For $\beta \rightarrow 0$ (infinite temperature), one obtains the Poisson eigenvalue statistics and for $\beta \rightarrow \infty$ (zero temperature), one gets the Clock eigenvalue statistics.

CMV matrices with independent random coefficients $\alpha_{n}$ are investigated in [17]; furthermore, each coefficient is assumed to have a rotationally invariant distribution. Associated to the random $n \times n$ CMV matrix $\mathcal{C}^{(n)}$ are $n$ random eigenvalues, which are denoted $e^{i \theta_{1}}, \ldots, e^{i \theta_{n}}$, with $\theta_{j} \in(-\pi, \pi]$. By rotation invariance, the average number of eigenvalues with $\theta_{j} \in[a, b)$ is $\frac{b-a}{2 \pi} n$. For this reason it is natural to rescale the eigenvalues by a factor of $n$. One can show that the rescaled eigenvalue process, that is, the random set of points $\left\{n \theta_{1}, \ldots, n \theta_{n}\right\} \subset \mathbb{R}$, converges to a point process. More precisely:

Theorem 2.3 (1.7 in [17]). (i) If $\mathbb{E}\left\{\left|\alpha_{k}\right|^{2}\right\}=o\left(k^{-1}\right)$, then the eigenvalue process converges to the clock process with spacing $2 \pi$, as $n \rightarrow \infty$.

(ii) If $\mathbb{E}\left\{\left|\alpha_{k}\right|^{2}\right\}-\frac{2}{\beta(k+1)}=o\left(k^{-1}\right)$ and $\mathbb{E}\left\{\log ^{2}\left[1-\left|\alpha_{k}\right|^{2}\right]\right\}=o\left(k^{-1}\right)$, then the eigenvalue process converges to the $\beta$-ensemble, as $n \rightarrow \infty$.

(iii) If $\mathbb{E}\left\{\left|\alpha_{k}\right|^{2}\right\} \geq(k+1)^{\epsilon-1}$ for some $\epsilon>0$ and $\mathbb{E}\left\{\left(1-\left|\alpha_{k}\right|^{2}\right)^{-s}\right\}=O(1)$ for some $s>0$, then the eigenvalue process converges to the Poisson process with intensity $\frac{1}{2 \pi} d x$, as $n \rightarrow \infty$.

This theorem shows that for rapidly decaying random coefficients, the eigenvalue statistics is Clock (which is the distribution corresponding to the "free" case). For slowly decaying random coefficients (including the i.i.d. cases studied before), the eigenvalue statistics is Poisson. In the critical case, the limit is given by the circular $\beta$ ensemble.

The circular $\beta$ ensemble with $n$ points $\mathrm{C} \beta \mathrm{E}_{n}$, is a point process on the unit circle. The total number of points is $n$ (non-random) and they are distributed so that

$$
\mathbb{E}_{n}^{\beta}(f)=\frac{1}{Z_{n, \beta}} \int_{-\pi}^{\pi} \ldots \int_{-\pi}^{\pi} f\left(e^{i \theta_{1}}, \ldots, e^{i \theta_{n}}\right)\left|\Delta\left(e^{i \theta_{1}}, \ldots, e^{i \theta_{n}}\right)\right|^{\beta} \frac{d \theta_{1}}{2 \pi} \cdots \frac{d \theta_{n}}{2 \pi}
$$


for any symmetric function $f$. Here, $Z_{n, \beta}$ is a normalizing constant and $\Delta\left(z_{1}, z_{2}, \ldots, z_{n}\right)=$ $\prod_{1 \leq j<k \leq n}\left(z_{k}-z_{j}\right)$ is the Vandermonde determinant. This is a standard family of ensembles in random matrix theory and was introduced by Dyson in [9]. This ensemble also arises as the Gibbs measure for $n$ identical charged particles confined to lie on the circle and interacting via the two-dimensional Coulomb law. For this reason, it is also known as the log-gas ([10]). As we explained earlier for eigenvalues of random CMV matrices, one may take the points of the $\mathrm{C} \beta \mathrm{E}_{n}$ and by unwrapping and rescaling obtain a sequence of point processes on the line. This sequence of point processes converges to what we call the circular $\beta$-ensemble and is the microscopic thermodynamic limit of the one-dimensional log-gas.

The proof of part (i) ("Clock") extends ideas from the non-random case obtained in [27]. Part (ii) ("circular $\beta$-ensemble") is proven using the exactly soluble example of our model found in [16] and an invariance (universality) principle that shows that the limiting eigenvalue statistics do not depend on the specifics of the probability distribution of the random coefficients. Part (iii) ("Poisson") is proven using the same road map described earlier in this section, and requires a different estimate of the decay of the fractional moments of the resolvent.

This theorem demonstrates that (at least for this model) the transition from Poisson to Clock statistics passes through a continuum of intermediate processes. This transition resembles the change in the eigenvalue statistics in a (microscopic) neighborhood of the (conjectured) metal/insulator transition in the multi-dimensional Anderson model.

Figures 1 and 2 can be used to visualise the transition in the distribution of the eigenvalues of CMV matrices with random decaying coefficients. Let us denote by $\left\{e^{i \theta_{1}(\omega)}, e^{i \theta_{2}(\omega)}, \ldots, e^{i \theta_{n}(\omega)}\right\}$ the eigenvalues of the random CMV matrix $\mathcal{C}^{(n)}(\omega)$ (the eigenvalues are listed in increasing order of their arguments: $\left.0 \leq \theta_{1}(\omega) \leq \theta_{2}(\omega) \leq \cdots \leq \theta_{n}(\omega)\right)$.

In Figure 1 we plot the eigenvalues of $\mathcal{C}^{(n)}(\omega)$ for $n=100$ in three situations: random coefficients with slow decay, critical decay, and fast decay. In the case of slow decay, we see clumps and gaps in the distribution of the eigenvalues, indicating Poisson distribution. In the case of fast decay, we see the clock distribution (strong repulsion between the eigenvalues, which implies equal spacings between consecutive eigenvalues, hence "clock" distribution). In the case of critical decay, we see weak repulsion between the eigenvalues.
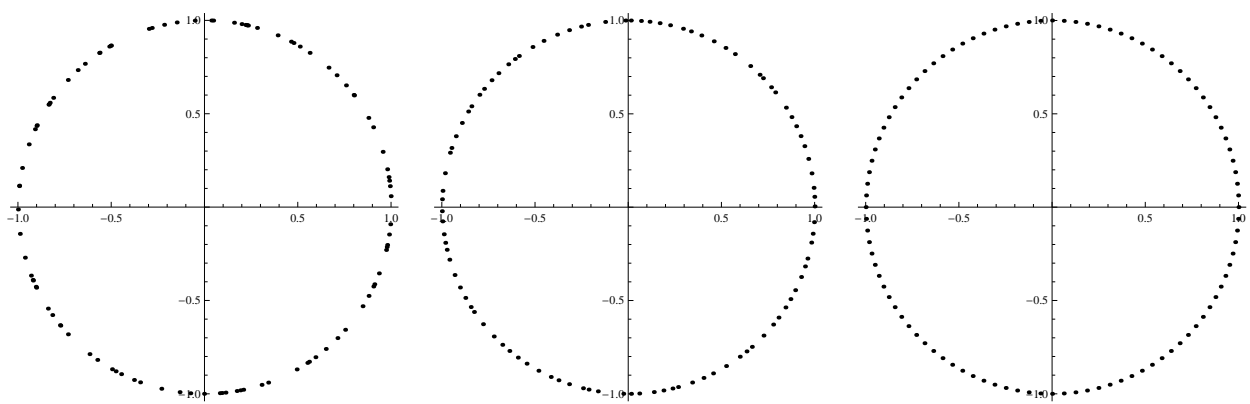

Figure 1. Transition in the eigenvalue statistics for CMV matrices with random decaying coefficients: from Poisson (left) to clock (right), via the $\mathrm{C} \beta \mathrm{E}$ (middle). We plot 100 points in each case.

Another way in which we can observe the transition in the distribution of the eigenvalues $\left\{e^{i \theta_{1}(\omega)}, e^{i \theta_{2}(\omega)}, \ldots, e^{i \theta_{n}(\omega)}\right\}$, of the matrix $\mathcal{C}^{(n)}(\omega)$ is to consider their arguments $\left\{\theta_{1}(\omega), \theta_{2}(\omega), \ldots, \theta_{n}(\omega)\right\}$ and investigate the list of consecutive differences (level spacings):

$$
\mathcal{L}(\omega)=\left\{\theta_{2}(\omega)-\theta_{1}(\omega), \theta_{3}(\omega)-\theta_{2}(\omega), \ldots, \theta_{n}(\omega)-\theta_{n-1}(\omega)\right\}
$$


In Figure 2 we plot histograms of the list $\mathcal{L}(\omega)$ for $n=1000$ in the three cases (random coefficients with slow decay, critical decay, and fast decay). The first histogram, corresponding to slow decay of the random coefficients exhibits the behavior of a decreasing exponential function, indicating a lack of repulsion between the eigenvalues. The third histogram, corresponding to fast decay of the random coefficients, indicates strong repulsion between the eigenvalues, while the second histogram interpolates between the two, indicating a weak repulsion between the eigenvalues.
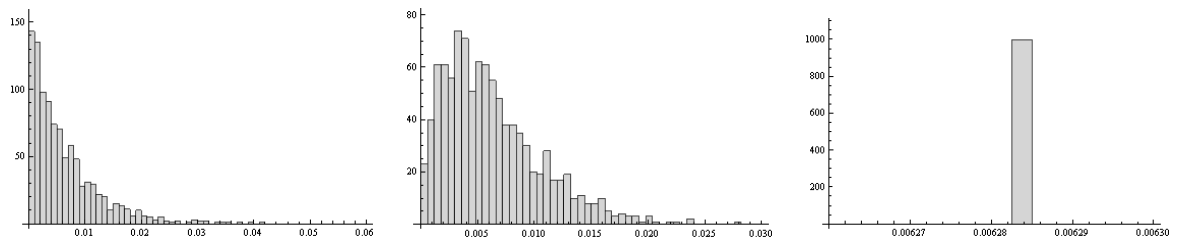

FiguRE 2. Transition in the level spacing statistics for CMV matrices with random decaying coefficients: from Poisson (left) to clock (right), via the $\mathrm{C} \beta \mathrm{E}$ (middle). We plot the histogram associated to 1000 eigenvalues in each case.

\section{Spectral Properties of CMV Matrices Associated to Measures Generated by McMullen's Three Circle Family}

This line of research is suggested by the fact that, in the known cases, the Poisson local eigenvalue statistics corresponds to a pure point spectral measure, the "Clock" local eigenvalue statistics corresponds to an absolutely continuous spectral measure, whereas critical eigenvalue statistics correspond to operators with singular continuous spectrum.

Let us consider the family $\left\{\mu_{\theta}\right\}, 0 \leq \theta \leq 2 \pi / 3$, of singular continuous measures on the unit circle generated by the McMullen's three circle family (or "symmetric pairs of pants") described in [18]. These measures are special cases of the Patterson-Sullivan measures constructed in hyperbolic geometry [23, 24, $31,32]$ and are supported on sets of fractional Hausdorff dimension. Hence, they interpolate between a pure point measure and an absolutely continuous measure on the unit circle, with the Hausdorff dimension of the support of the measure changing continuously from 0 to 1 . These measures and some spectral properties of the associated CMV operators are investigated in [12].

The measures generated by McMullen's three circle family from [18] are obtained as follows: for every $\theta$, with $0<\theta<2 \pi / 3$, let $C_{1}, C_{2}, C_{3}$ be three circles of equal radii, arranged symmetrically around the unit circle $\mathbb{T}$ and meeting $\mathbb{T}$ in an arc of length $\theta$. Each $C_{i}=C_{i}(\theta)$ has radius $r=r(\theta)=\tan \frac{\theta}{2}$. The circle centers are at $z_{0}=a, z_{1}=a e^{2 \pi i / 3}$, and $z_{2}=a e^{-2 \pi i / 3}$, where $a=a(\theta)=\sec \frac{\theta}{2}$. Consider also the reflection $\rho_{i}: \mathbb{C} \rightarrow \mathbb{C}$ in the circle $C_{i}$ :

$$
\rho_{i}(z)=\frac{r^{2}}{\bar{z}-\bar{z}_{i}}+z_{i}
$$

Let $\Gamma_{\theta}$ be the group generated by these three reflections, $\Lambda_{\theta}$ the associated limit set and $\mu_{\theta}$ to corresponding limiting measure (supported on the set $\Lambda_{\theta}$ ). As defined in [18] and [12], the measure $\mu_{\theta}$ is the unique probability measure $\mu$ supported on $\Lambda_{\theta} \subset \mathbb{T}$ with the property that

$$
\mu(\gamma(A))=\int_{A}\left|\gamma^{\prime}(z)\right|^{\delta} d \mu
$$

for any Lebesgue measurable set $A$ and any $\gamma \in \Gamma_{\theta}$, where $\delta$ is the Hausdorff dimension of the set $\Lambda_{\theta}$ and $\gamma^{\prime}$ denotes the ordinary derivative of the map $\gamma: \mathbb{T} \rightarrow \mathbb{T}$ with respect to the spherical metric (i.e., angle metric in radians). This means that $\mu_{\theta}$ behaves like a $\delta$-dimensional Hausdorff measure. 


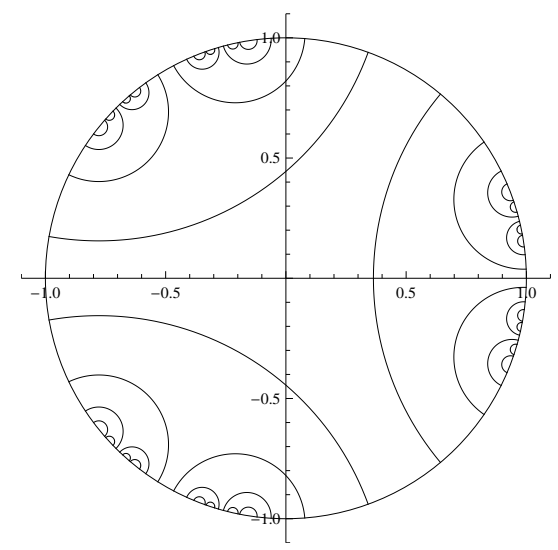

FigurE 3. The reflection group generated by McMullen's three circle family for $\theta=\frac{5 \pi}{9}$.

As $\theta$ changes from 0 to $2 \pi / 3$, the Hausdorff dimension of $\Lambda_{\theta}$ changes from 0 to 1 , as one can see in the following table:

\begin{tabular}{c|c}
\hline Angle $\theta$ (in degrees) & Hausdorff dimension of $\Lambda_{\theta}$ \\
\hline 0 & 0.00000000000000000000000000000 \\
\hline 10 & 0.11600944778617663747082865262 \\
\hline 20 & 0.15118368203822075169122305922 \\
\hline 30 & 0.18398306124833918694117818431 \\
\hline 40 & 0.21776581025463001217722392393 \\
\hline 50 & 0.25438289832389822256892055021 \\
\hline 60 & 0.29554647988904080826448492725 \\
\hline 70 & 0.34333399783359269399961502109 \\
\hline 80 & 0.40067612755882685166614065221 \\
\hline 90 & 0.47218912788210556616394926496 \\
\hline 100 & 0.56609805081992862445555013291 \\
\hline 110 & 0.70055063165213127375015834183 \\
\hline 120 & 1.00000000000000000000000000000
\end{tabular}

This table is obtained in [12] using a refinement of the algorithm for the computation of dimension from [18]. One can investigate the spectral properties of the unitary operators $\mathcal{C}_{\theta}$ associated to the measures $\mu_{\theta}$ for $0<\theta<2 \pi / 3$; of particular interest are the moments of $\mu_{\theta}$ and the transition in the eigenvalue distribution as $\theta$ changes from 0 to $2 \pi / 3$. Note that, for any $\theta$, the measure $\mu_{\theta}$ is the spectral measure of the unitary operator $\mathcal{C}_{\theta}$.

The spectral properties of the CMV operator $\mathcal{C}_{\theta}$ and of its finite truncations $\mathcal{C}_{\theta}^{(n)}$ can be investigated with the help of the moments

$$
c_{k}^{(\theta)}=\int_{\mathbb{T}} z^{-k} d \mu_{\theta}
$$

of the measure $\mu_{\theta}$ (as before, $\mathbb{T}$ denotes the unit circle).

The symmetries of the underlying system of generators imply that $c_{k}^{(\theta)}=c_{-k}^{(\theta)} \in \mathbb{R}$, and that only every third moment is non-zero. These moments encapsulate much of the information contained in $\mu_{\theta}$; for example, knowledge of them is sufficient to "quantize" the classical dynamics of hyperbolic reflections to the unitary $\mathrm{CMV}$ matrices $\mathcal{C}_{\theta}$.

A result from [12] shows that McMullen's algorithm can be used to construct a family of atomic measures $\mu_{\theta}^{(\varepsilon)}$ which approximate the measure $\mu_{\theta}$. More precisely, for any $\varepsilon>0$, one can find a positive 
integer $M(\varepsilon)$, a set of points $z_{0}^{(\varepsilon)}, z_{1}^{(\varepsilon)}, \ldots, z_{M(\varepsilon)}^{(\varepsilon)}$ on the unit circle and a set of weights $w_{0}^{(\varepsilon)}, w_{1}^{(\varepsilon)}, \ldots, w_{M(\varepsilon)}^{(\varepsilon)}$, such that the atomic measure

$$
\mu_{\theta}^{(\varepsilon)}=\sum_{l=0}^{M(\varepsilon)} w_{l}^{(\varepsilon)} \delta_{z_{l}^{(\varepsilon)}}
$$

is a probability measure and $\mu_{\theta}^{(\varepsilon)}$ converges weakly to $\mu_{\theta}$, as $\varepsilon \rightarrow 0$.

One can obtain approximations for the moments of $\mu_{\theta}$ by computing the moments of the atomic measure $\mu_{\theta}^{(\varepsilon)}$ for small enough $\varepsilon>0$. Thus, one gets:

Theorem 3.1 (3.2 in [12]). The moments $c_{k}^{(\theta)}$ of the measure $\mu_{\theta}$ satisfy

$$
c_{3 j-1}^{(\theta)}=c_{3 j+1}^{(\theta)}=0
$$

and

$$
c_{3 j}^{(\theta)}=(-1)^{j} \lim _{\varepsilon \rightarrow 0} \sum_{l=0}^{M(\varepsilon) / 6} 6 m_{l}^{(\varepsilon)} \cos \left[3 j \phi_{l}^{(\varepsilon)}\right] .
$$

where $M(\varepsilon), m_{l}^{(\varepsilon)}$, and $\phi_{l}^{(\varepsilon)}$ are quantities given by McMullen's algorithm.

The previous theorem can be used to perform an efficient numerical analysis of the orthogonal polynomials associated to the measure $\mu$ and of the spectrum of the truncated CMV matrices $\mathcal{C}_{\theta}^{(n)}$ (since the orthogonal polynomials, the Verblunsky coefficients, and the CMV matrices can be constructed starting from the moments). One can see that the eigenvalue distribution of $\mathcal{C}_{\theta}$ converges to "clock" as $\theta \rightarrow 2 \pi / 3$.

Therefore, as one can observe in Figure 4, there is a transition in the distribution of the eigenvalues from Three Points $(\theta=0)$ to "Clock" $(\theta=2 \pi / 3)$, while the corresponding spectral measures are changing from an atomic measure $(\theta=0)$ to Lebesgue $(\theta=2 \pi / 3)$ passing through the continuum of singular continuous measures $\mu_{\theta}$.
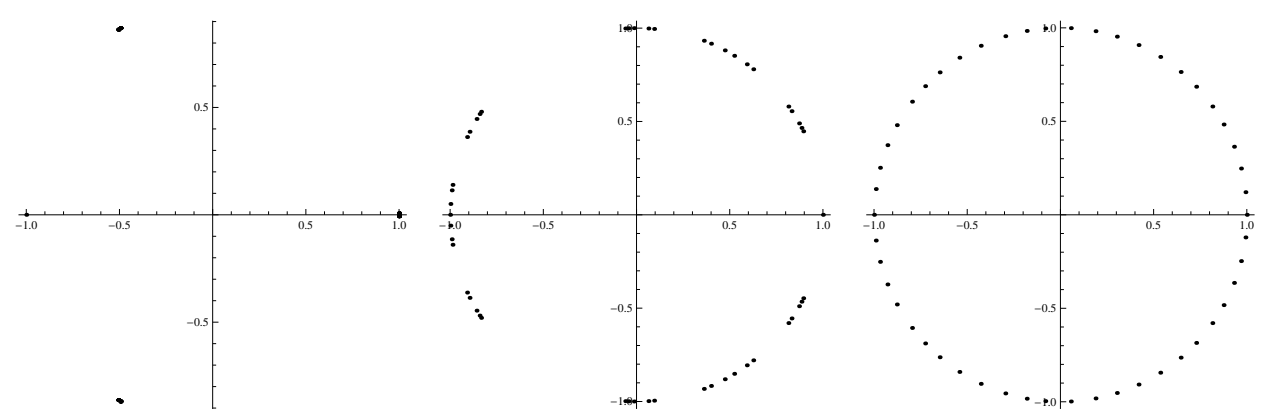

Figure 4. Plots of the eigenvalues of the CMV matrix $\mathcal{C}_{\theta}$ for $\theta=\frac{\pi}{18}$ (left), $\theta=\frac{5 \pi}{9}$ (middle), and $\theta=\frac{119 \pi}{180}$ (right).

As explained in the previous section, another way in which we can observe the transition in the distribution of the eigenvalues $\left\{e^{i \theta_{1}(\theta)}, e^{i \theta_{2}(\theta)}, \ldots, e^{i \theta_{n}(\theta)}\right\}$, of the matrix $\mathcal{C}_{\theta}^{(n)}$ is to consider their arguments $\left\{\theta_{1}(\theta), \theta_{2}(\theta), \ldots, \theta_{n}(\theta)\right\}$ (listed in increasing order) and investigate the list of consecutive differences:

$$
\mathcal{L}(\theta)=\left\{\theta_{2}(\theta)-\theta_{1}(\theta), \theta_{3}(\theta)-\theta_{2}(\theta), \ldots, \theta_{n}(\theta)-\theta_{n-1}(\theta)\right\}
$$

In Figure 5 we plot histograms of the list $\mathcal{L}(\theta)$ in the three cases: $\theta=\frac{\pi}{18}, \theta=\frac{5 \pi}{9}$, and $\theta=\frac{119 \pi}{180}$. As expected, the first histogram indicates the fact that, for small $\theta$, the eigenvalues are clustered around the 

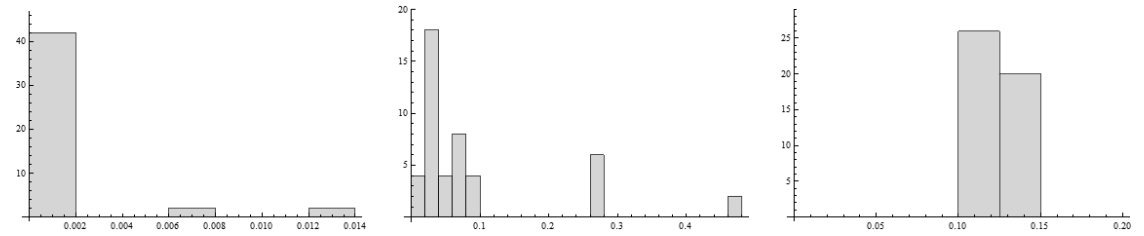

FiguRE 5. Transition in the distribution of the spacings of the eigenvalues of finite truncations of the matrix $\mathcal{C}_{\theta}$ for $\theta=\frac{\pi}{18}$ (left), $\theta=\frac{5 \pi}{9}$ (middle), and $\theta=\frac{119 \pi}{180}$ (right).

three cube roots of unity. The third histogram indicates a strong repulsion of the eigenvalues (close to the "clock distribution"), while the second histogram interpolates between the two.

It should be mentioned that the spectral analysis of operators with singular continuous spectral measures is in general very difficult. The approach used in [12] relies heavily on the nice properties of the measure $\mu_{\theta}$ (especially the many symmetries in its structure given by the hyperbolic reflections).

The results presented in Sections 2 and 3 suggest that, generically, the clock distribution of the eigenvalues (which indicates a strong repulsion between the eigenvalues) corresponds to operators with spectral measures that are absolutely continuous with respect to the Lebesgue measure. As explained in Section 2, Anderson localization indicates that if there is enough "randomness" in the coefficients of an operator, then its spectral measure is pure point and the distribution of its eigenvalues is Poisson. This leaves the operators with singular continuous spectral measures in the middle, indicating that these operators will have interesting eigenvalue distributions.

\section{References}

[1] M. Aizenman, S. Molchanov. Localization at large disorder and at extreme energies: an elementary derivation. Comm. Math. Phys., 157 (1993), 245-278.

[2] M. Aizenman, J. Schenker, R. Friedrich, D. Hundertmark. Finite-volume fractional moment criteria for Anderson localization. Commun. Math. Phys., 224 (2001), 219-253.

[3] P.W. Anderson. Absence of diffusion in certain random lattices. Phys. Rev., 109 (1958), 1492-1505.

[4] J. Bellissard, P. Hislop, G. Stolz. Correlation estimates in the Anderson model. J. Stat. Phys., 129 (2007), no. 4, 649-662.

[5] J. Bourgain, C. Kenig. On localization in the continuous Anderson-Bernoulli model in higher dimension. Invent. Math., 161 (2005), 389-426.

[6] J-M. Combes, F. Germinet, A. Klein. Generalized eigenvalue-counting estimates for the Anderson model. J. Stat. Phys., 135 (2009), no. 2, 201-216.

[7] M.J. Cantero, L. Moral, L. Velázquez. Five-diagonal matrices and zeros of orthogonal polynomials on the unit circle. Linear Algebra Appl., 362 (2003), 29-56.

[8] R. del Rio, S. Jitomirskaya, Y. Last, B. Simon. What is localization? Phys. Rev. Lett., 75 (1995), $117-119$.

[9] F.J. Dyson. Statistical theory of the energy levels of complex systems. I, II, and III. J. Math. Phys., 3 (1962), 140-156, 157-165, and 166-175.

[10] P.J. Forrester. Log-gases and Random matrices. London Mathematical Society Monographs Series, 34. Princeton University Press, Princeton, NJ, 2010.

[11] J. Fröhlich, T. Spencer. Absence of diffusion in the Anderson tight binding model for large disorder or low energy. Comm. Math. Phys., 88 (1983), no. 2, 151-184.

[12] K. Gittins, N. Peyerimhoff, M. Stoiciu, D. Wirosoetisno. Some spectral applications of McMullen's Hausdorff dimension algorithm. Conform. Geom. Dyn., 16 (2012), 184-203.

[13] I. Ja. Goldsheid, S.A. Molchanov, L.A. Pastur. A random homogeneous Schrödinger operator has a pure point spectrum. Funkcional. Anal. i Priložen., 11 (1977), no. 1, 1-10, 96.

[14] G.M. Graf, A. Vaghi. A remark on the estimate of a determinant by Minami. Lett. Math. Phys., 79 (2007), no. 1, $17-22$.

[15] A. Klein, O. Lenoble, P. Müller. On Mott's formula for the ac-conductivity in the Anderson model. Ann. of Math., 166 (2007), no. 2, 549-577.

[16] R. Killip, I. Nenciu. Matrix models for circular ensembles. Int. Math. Res. Not. (2004), no. 50, $2665-2701$.

[17] R. Killip, M. Stoiciu. Eigenvalue Statistics for CMV Matrices: From Poisson to Clock via Random Matrix Ensembles. Duke Math. J., 146 (2009), no. 3, 361-399. 
[18] C.T. McMullen. Hausdorff dimension and conformal dynamics. III. Computation of dimension. Amer. J. Math., 120 (1998), no. 4, 691-721.

[19] M.L. Mehta. Random matrices. Third Edition. Pure and Applied Mathematics (Amsterdam), 142. Elsevier/Academic Press, Amsterdam, 2004.

[20] N. Minami. Local fluctuation of the spectrum of a multidimensional Anderson tight-binding model. Comm. Math. Phys., 177 (1996), 709-725.

[21] S. Molchanov. Structure of the eigenfunctions of one-dimensional unordered structures. (Russian) Izv. Akad. Nauk SSSR Ser. Mat., 42 (1978), no. 1, 70-103, 214.

[22] S. Molchanov. The local structure of the spectrum of the one-dimensional Schrödinger operator. Comm. Math. Phys., 78 (1981), 429-446.

[23] P.J. Nicholls. A measure on the limit set of a discrete group. In Ergodic theory, symbolic dynamics, and hyperbolic spaces. Edited by T. Bedford, M. Keane, C. Series. Oxford Science Publications. The Clarendon Press, Oxford University Press, New York, 1991.

[24] S.J. Patterson. The limit set of a Fuchsian group. Acta Math., 136 (1976), no. 3-4, 241-273.

[25] B. Simon. Orthogonal Polynomials on the Unit Circle, Vol. 1. American Mathematical Society Colloquium Publications. American Mathematical Society, Providence, Rhode Island, 2004.

[26] B. Simon. Orthogonal Polynomials on the Unit Circle, Vol. 2. American Mathematical Society Colloquium Publications. American Mathematical Society, Providence, Rhode Island, 2004.

[27] B. Simon. Fine structure of the zeros of orthogonal polynomials. I. A tale of two pictures. Electron. Trans. Numer. Anal., 25 (2006), 328-368 (electronic).

[28] B. Simon. CMV matrices: five years after. J. Comput. Appl. Math., 208 (2007), no. 1, 120-154.

[29] M. Stoiciu. The statistical distribution of the zeros of random paraorthogonal polynomials on the unit circle. J. Approx. Theory, 139 (2006), 29-64.

[30] M. Stoiciu. Poisson Statistics for Eigenvalues: From Random Schrödinger Operators to Random CMV Matrices. CRM Proceedings and Lecture Notes, volume 42 (2007), 465-475.

[31] D. Sullivan. The density at infinity of a discrete group of hyperbolic motions. Inst. Hautes Études Sci. Publ. Math. No., 50 (1979), 171-202.

[32] D. Sullivan. Entropy, Hausdorff measures old and new, and limit sets of geometrically finite Kleinian groups. Acta Math., 153 (1984), no. 3-4, 259-277. 